\title{
Effect of Hexamethonium on the Augmented Adrenaline Secretion of the Adrenal Gland Causable by Insulin Hypoglycemia
}

\author{
By \\ Kazukuni Yamashita \\ (山下 一 邦) \\ From the Department of Physiology, Nagasaki University \\ School of Medicine, Nagasaki \\ (Received for publication, November 12, 1956)
}

In view of the blocking effect of hexamethonium on the autonomic ganglia it is deemed desirable to ascertain whether the adrenal medulla is also paralysed by hexamethonium.

The previously reported work ${ }^{1)}$ provided evidence that the acceleration of adrenaline secretion causable by acetylcholine was definitely suppressed by hexamethonium. Following up these acetylcholine experiments, a further attempt was made in the present investigation to know how hexamethonium affects the augmented adrenaline secretion causable by insulin hypoglycemia.

Some years ago, Schachter') demonstrated in dogs that an application of insulin followed by hexamethonium produced more intensive hypoglycemia than that without hexamethonium. From these results it was deduced by him that hexamethonium possessed the paralysing action on the adrenal medulla and suppressed the augmentation of adrenaline secretion causable by insulin hypoglycemia. The evidence presented by him is, however, an indirect one. In the present investigation experiments were made in estimating directly the rate of adrenaline secretion of the adrenal.

\section{EXPERIMENTAL}

\section{Methods}

Dogs (8.1-14.8 kg. in weight), anesthetized with evipan-sodium, were experimented on. For collecting the adrenal venous blood, the lumbar route preparation of Satake $e t a l^{3}$ ) was applied. The blood pressure was recorded by a mercury manometer connected with the femoral artery. The blood sugar content was estimated by the method of Hagedorn and Jensen. 
Five to 15 minutes before insulin injection, the initial adrenal blood specimens were collected. Then, insulin was injected intravenously in a dose of 7 units per kg. of body weight. Thirty, 60, 70, 80 and 88 minutes after insulin the adrenal venous blood was collected. Ninety minutes after insulin, hexamethonium (Bistrium bromide, Squibb) was given intravenously in a dose of $0.75 \mathrm{mg}$. per $\mathrm{kg}$. Thereafter the adrenal blood was sampled at intervals. The adrenaline content of the sampled adrenal venous blood specimens was estimated colorimetrically by the Bloor \& Bullen's arseno-molybdic acid method,") adrenaline (Sankyo Co.) being taken as the standard.

\section{Results AND Discussion}

The data are shown in table $I$.

As the control experiments, insulin alone was applied in 3 dogs without injecting hexamethonium.

In Exp. 1, the blood sugar content, which was $0.106-0.107 \%$ before insulin, decreased remarkably after insulin. It was determined as $0.072 \%$ 30 minutes after insulin application and $0.064 \% 60$ minutes. Thereafter it decreased further and 150 minutes after insulin it reached $0.041 \%$.

The initial adrenaline secretion rate in this dog was too small to be estimated. Thirty minutes after insulin injection the rate of adrenaline secretion was found to have increased to $0.15 \mu \mathrm{g}$. per $\mathrm{kg}$. per minute, 30 minutes later it increased further and $0.21 \mu \mathrm{g}$. was reached. Then it was measured as $0.24 \mu \mathrm{g}$. 70 minutes after insulin, $0.26 \mu \mathrm{g}$. 80 minutes, 0.25 $\mu \mathrm{g} .88$ minutes, $0.27 \mu \mathrm{g} .100$ minutes, $0.21 \mu \mathrm{g}$. 110 minutes, $0.16 \mu \mathrm{g} .120$ minutes and $0.19 \mu \mathrm{g} .150$ minites, respectively.

A similar result was obtained in Exp. 2.

The blood sugar content before insulin was $0.111 \%$. It decreased markedly after insulin and was measured as $0.071 \% 30$ minutes after insulin, $0.066 \% 60$ minutes, $0.064 \% 88$ minutes, $0.066 \% 120$ minutes and $0.072 \% 150$ minutes.

The adrenaline secretion rate before insulin was immeasurably small. After insulin, the rate of secretion was found to increase and was determined as $0.05 \mu \mathrm{g}$. per $\mathrm{kg}$. per minute 30 minutes after injection, $0.07 \mu \mathrm{g}$. 60 minutes, $0.09 \mu \mathrm{g}$. 80 minutes, $0.10 \mu \mathrm{g}$. 88minutes, $0.12 \mu \mathrm{g}$. 100,110 and 120 minutes and $0.03 \mu \mathrm{g} .150$ minutes, respectively.

In Exp. 3, the blood sugar content decreased from $0.106-0.110 \%$ and 30 minutes after insulin it reached $0.061 \%$. Thereafter it decreased gradually and was measured as $0.054 \% 60$ minutes after insulin, $0.051 \%$ 88 minutes, and $0.049 \% 115$ minutes, respectively.

The initial rate of adrenaline secretion in this dog was $0.04 \mu \mathrm{g}$. per $\mathrm{kg}$. per minute. It increased and reached $0.14 \mu \mathrm{g} .30$ minutes after in- 
sulin injection. It was measured as $0.10 \mu \mathrm{g} .60$ minutes after insulin, 0.12 $\mu$ g. 70 minutes, $0.15 \mu$ g. 80 minutes, $0.25 \mu \mathrm{g}$. 88 minutes, $0.21 \mu \mathrm{g} .100$ minutes and $0.13 \mu \mathrm{g} .115$ minutes, respectively.

The hexamethonium-experiemnts were performed in 4 dogs.

In Exp. 4, the blood sugar content before insulin was $0.119-0.120 \%$. It was reduced by insulin injection and 60-88 minutes after injection it was determined as $0.060-0.064 \%$. Then hexamethonium was given. After hexamethonium blood sugar content increased only slightly.

The initial adrenaline secretion rate was in this experiment $0.02 \mu \mathrm{g}$. per $\mathrm{kg}$. per minute. After insulin it increased. It reached $0.13 \mu \mathrm{g} .60$ minutes after insulin and it was measured as $0.17 \mu \mathrm{g} ., 0.38 \mu \mathrm{g}$. and 0.30 $\mu$ g. 70,80 and 88 minutes after insulin, respectively. Ninety minutes after insulin, hexamethonium was injected. Then the rate of adrenaline secretion was found to have decreased remarkably. It was estimated to be $0.08 \mu \mathrm{g} .5$ minutes after hexamethonium, $0.04 \mu \mathrm{g}$. 10 minutes, $0.03 \mu \mathrm{g}$. 20 minutes and $0.01 \mu \mathrm{g} .60$ minutes.

In Exp. 5, the blood sugar content before insulin was $0.115 \%$. Thirty, 60 and 80 minites after insulin it was determined as $0.063 \%, 0.058 \%$ and $0.054 \%$, respectively. Then, hexamethonium was injected. The blood sugar content was gradually decreased.

Before insulin, the adrenaline secretion rate was $0.01 \mu \mathrm{g}$. per $\mathrm{kg}$. per minute. Thirty minutes after insulin it was increased and measured as $0.09 \mu \mathrm{g}$. Sixty, 70, 80 and 88 minutes after insulin it was found to be $0.13 \mu \mathrm{g}$., $0.28 \mu \mathrm{g}$., $0.17 \mu \mathrm{g}$. and $0.13 \mu \mathrm{g}$., respectively. Hexamethonium was given 90 minutes after insulin. On receiving hexamethonium the rate of secretion was decreased markedly and was measured as $0.02 \mu \mathrm{g}$. per $\mathrm{kg}$. per minute 10 minutes after hexamethonium, immeasurably small 20 minutes and $0.006 \mu \mathrm{g} .50$ minutes, respectively.

In Exp. 6, the blood sugar content was reduced from $0.123-0.124 \%$ to $0.060 \%$ within 30 minutes after insulin. From 60 to 80 minutes after insulin it was $0.054 \%$. Then the injection of hexamethonium was made. The blood sugar decreased further and reached $0.048 \% 140$ minutes after insulin.

The basal rate of adrenaline secretion in this experiment was 0.05$0.06 \mu \mathrm{g}$. per kg. per minute. After insulin the rate of secretion was found to increase and was determined to be $0.23 \mu \mathrm{g}$. per $\mathrm{kg}$. per minute 30 minutes, $0.21 \mu \mathrm{g}$. 60 minutes, $0.23 \mu \mathrm{g}$. 70 minutes, $0.22 \mu \mathrm{g}$. 80 minutes and $0.25 \mu \mathrm{g}$. 88 minutes after insulin. Ninety-five minutes after insulin application, hexamethonium was injected. The rate of secretion then decreased remarkably and it was $0.03 \mu \mathrm{g}$. per $\mathrm{kg}$. per minute 10 minutes after hexamethonium, $0.02 \mu \mathrm{g} .30$ and 45 minutes.

In Exp. 7, the blood sugar content decreased from $0.112-0.114 \%$ to 
TABLE I

The Adrenaline Secretion Rate after Insulin with and without Hexamethonium

\begin{tabular}{|c|c|c|c|c|c|c|c|c|c|c|c|c|}
\hline 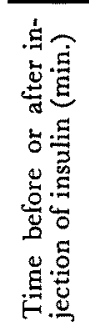 & 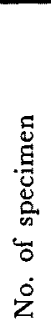 & 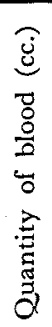 & 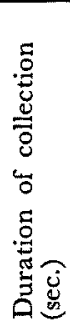 & 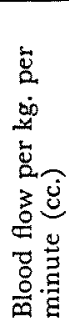 & 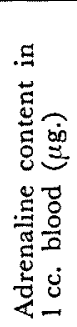 & 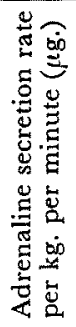 & 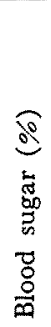 & 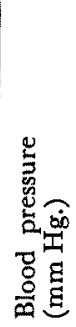 & 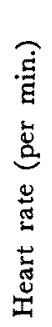 & 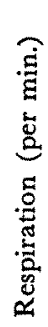 & 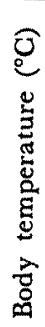 & 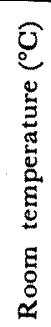 \\
\hline
\end{tabular}

(A) Insulin

Exp. 1. Dog $14.8 \mathrm{~kg}$. 우

before

\begin{tabular}{r|l|l|l|l|l|l|l|l|l|l|l|l|}
15 & 1 & 3.8 & 30 & 0.51 & $*$ & & 0.107 & 140 & 160 & 23 & 39.0 & 14 \\
5 & 2 & 4.0 & 30 & 0.54 & $*$ & & 0.106 & 136 & 136 & 25 & 38.9 & 14
\end{tabular}

Intravenous injection of insulin (7 units per $\mathrm{kg}$.)

after

\begin{tabular}{r|r|l|l|l|l|l|l|l|l|l|r|r}
30 & 3 & 6.4 & 30 & 0.87 & 0.17 & 0.15 & 0.072 & 132 & 156 & 37 & 39.0 & 14.5 \\
60 & 4 & 4.1 & 20 & 0.83 & 0.25 & 0.21 & 0.064 & 114 & 160 & 40 & 39.0 & 14.5 \\
70 & 5 & 3.0 & 20 & 0.61 & 0.39 & 0.24 & 0.059 & 106 & 166 & 43 & 39.1 & 14.5 \\
80 & 6 & 3.0 & 20 & 0.61 & 0.42 & 0.26 & 0.055 & 128 & 170 & 36 & 39.1 & 14.5 \\
88 & 7 & 3.1 & 20 & 0.63 & 0.39 & 0.25 & 0.054 & 106 & 175 & 40 & 39.1 & 14.5 \\
100 & 8 & 3.4 & 20 & 0.69 & 0.38 & 0.27 & 0.047 & 110 & 194 & 36 & 38.9 & 14.5 \\
110 & 9 & 3.4 & 20 & 0.69 & 0.30 & 0.21 & 0.045 & 112 & 198 & 36 & 38.9 & 14.5 \\
120 & 10 & 3.4 & 20 & 0.69 & 0.23 & 0.16 & 0.045 & 110 & 200 & 36 & 38.7 & 14.5 \\
150 & 11 & 3.3 & 20 & 0.67 & 0.28 & 0.19 & 0.041 & 106 & 165 & 22 & 38.8 & 14.5
\end{tabular}

Exp. 2. Dog $13.3 \mathrm{~kg}$. $\delta$

before

$$
\begin{aligned}
& \begin{array}{r|l|l|l|l|l|l|l|l|l|l|l|l}
15 & 1 & 1.7 & 30 & 0.26 & * & & 0.111 & 138 & 130 & 52 & 38.8 & 19 \\
5 & 2 & 1.7 & 30 & 0.26 & * & & 0.111 & 136 & 148 & 58 & 38.8 & 19
\end{array} \\
& \text { Intravenous injection of insulin (7 units per } \mathrm{kg} \text {.) }
\end{aligned}
$$

after

\begin{tabular}{r|r|r|r|r|r|r|r|r|r|r|r|r}
30 & 3 & 1.9 & 30 & 0.29 & 0.18 & 0.05 & 0.071 & 122 & 150 & 70 & 38.1 & 19 \\
60 & 4 & 1.7 & 30 & 0.26 & 0.27 & 0.07 & 0.066 & 122 & 146 & 66 & 37.9 & 19 \\
70 & 5 & 1.3 & 30 & 0.20 & 0.30 & 0.06 & 0.064 & 116 & 148 & 68 & 37.8 & 19 \\
80 & 6 & 2.1 & 30 & 0.32 & 0.28 & 0.09 & 0.064 & 118 & 150 & 52 & 37.4 & 19 \\
88 & 7 & 2.2 & 30 & 0.33 & 0.31 & 0.10 & 0.064 & 108 & 142 & 62 & 37.5 & 19 \\
100 & 8 & 1.8 & 30 & 0.27 & 0.43 & 0.12 & & 106 & 146 & 56 & 37.4 & 19 \\
110 & 9 & 1.8 & 30 & 0.27 & 0.46 & 0.12 & 0.067 & 108 & 150 & 58 & 37.5 & 19 \\
120 & 10 & 2.0 & 30 & 0.30 & 0.41 & 0.12 & 0.066 & 104 & 148 & 52 & 37.5 & 19 \\
150 & 11 & 1.6 & 30 & 0.24 & 0.14 & 0.03 & 0.072 & 110 & 158 & 48 & 37.4 & 19
\end{tabular}

Exp. 3. Dog $8.1 \mathrm{~kg}$. $\quad$ ?

before

$$
\begin{array}{r|l|l|l|l|l|l|l|l|l|l|l|l}
15 & 1 & 2.0 & 30 & 0.49 & 0.08 & 0.04 & 0.106 & 116 & 170 & 20 & 38.3 & 15.5 \\
5 & 2 & 1.8 & 30 & 0.44 & 0.09 & 0.04 & 0.110 & 122 & 158 & 22 & 38.1 & 15.5 \\
\text { Intravenous injection of insulin (7 units per kg.) }
\end{array}
$$

after

\begin{tabular}{r|r|r|r|r|r|r|r|r|r|r|r|r}
30 & 3 & 1.6 & 30 & 0.40 & 0.36 & 0.14 & 0.061 & 110 & 135 & 23 & 37.8 & 15.5 \\
60 & 4 & 2.5 & 60 & 0.31 & 0.33 & 0.10 & 0.054 & 102 & 132 & 20 & 37.8 & 15.5 \\
70 & 5 & 2.2 & 60 & 0.27 & 0.43 & 0.12 & 0.053 & 104 & 148 & 22 & 37.2 & 15.5 \\
80 & 6 & 1.4 & 30 & 0.35 & 0.42 & 0.15 & 0.051 & 104 & 165 & 21 & 37.1 & 15.5 \\
88 & 7 & 1.3 & 30 & 0.32 & 0.77 & 0.25 & 0.051 & 100 & 160 & 25 & 37.0 & 15.5 \\
100 & 8 & 1.3 & 30 & 0.32 & 0.67 & 0.21 & 0.049 & 102 & 165 & 24 & 36.9 & 15.5 \\
115 & 9 & 1.7 & 60 & 0.21 & 0.61 & 0.13 & 0.049 & 98 & 164 & 18 & 36.9 & 15.5
\end{tabular}


(B) Insulin-Hexamethonium

before

Exp. 4. Dog $9.4 \mathrm{~kg}$. ิ

\begin{tabular}{|c|c|c|c|c|c|c|c|c|c|c|c|c|}
\hline $\begin{array}{r}10 \\
5\end{array}$ & $\begin{array}{l}1 \\
2\end{array}$ & $\begin{array}{l}1.8 \\
1.7\end{array}$ & $\begin{array}{l}30 \\
30\end{array}$ & $\begin{array}{l}0.37 \\
0.35\end{array}$ & $\begin{array}{l}0.06 \\
0.06\end{array}$ & $\begin{array}{l}0.02 \\
0.02\end{array}$ & $\begin{array}{l}0.120 \\
0.119\end{array}$ & $\begin{array}{l}154 \\
152\end{array}$ & $\begin{array}{l}220 \\
185\end{array}$ & $\begin{array}{l}26 \\
20\end{array}$ & $\begin{array}{l}37.8 \\
37.7\end{array}$ & $\begin{array}{l}12.5 \\
12.5\end{array}$ \\
\hline \multicolumn{13}{|c|}{ Intravenous injection of insulin ( 7 units per kg.) } \\
\hline 30 & 3 & 18 & 30 & 037 & ( 24 & $0 \cap 0$ & 0110 & 140 & & & & \\
\hline $\begin{array}{l}30 \\
60\end{array}$ & $\begin{array}{l}3 \\
4\end{array}$ & 1.0 & $\begin{array}{l}30 \\
30\end{array}$ & 0.41 & $\begin{array}{l}0.24 \\
0.31\end{array}$ & 0.09 & 0.112 & $\begin{array}{l}140 \\
132\end{array}$ & 203 & 26 & 37.3 & 12 \\
\hline 70 & 5 & 2.0 & 30 & 0.43 & 0.40 & 0.17 & 0.060 & 132 & 215 & 25 & 37.5 & 12 \\
\hline 80 & 6 & 2.5 & 30 & 0.53 & 0.72 & 0.38 & 0.062 & 126 & 20 & 18 & 37.5 & 12 \\
\hline 88 & 7 & 2.7 & 30 & 0.57 & 0.52 & 0.30 & 0.064 & 130 & 205 & 22 & 378 & 12 \\
\hline 90 & \multicolumn{12}{|c|}{ Intravenous injection of hexamethonium ( $0.75 \mathrm{mg}$. per kg.) } \\
\hline 95 & 8 & 1.7 & 30 & 0.35 & 0.22 & 0.08 & 0.068 & 90 & 144 & 18 & 37.2 & 11.5 \\
\hline 100 & 9 & 1.5 & 30 & 0.33 & 0.11 & 0.04 & & 92 & 13 & 16 & 36 & 11.5 \\
\hline 110 & 10 & 1.3 & 30 & 0.28 & 0.09 & 0.03 & & 78 & 13 & 20 & 36.9 & .5 \\
\hline 120 & 11 & 1.8 & 30 & 0.37 & 0.13 & 0.04 & 0.071 & 76 & 138 & 15 & 36.5 & 1.5 \\
\hline 150 & 12 & 1.7 & 30 & 0.35 & 0.03 & 0.01 & 0.075 & 80 & 140 & 17 & 35.9 & 11.5 \\
\hline
\end{tabular}

Exp. 5. Dog $9.5 \mathrm{~kg}$. $\delta$

before

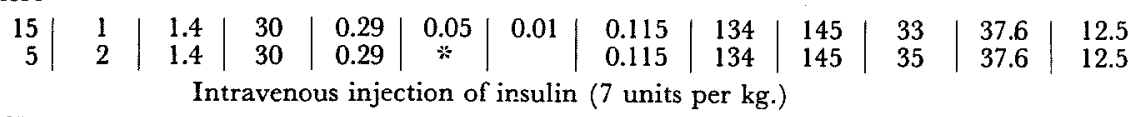

after

Intravenous injection of insulin ( 7 units per $\mathrm{kg}$.)

\begin{tabular}{r|r|l|l|l|l|l|l|l|l|l|l|l}
30 & 3 & 1.7 & 30 & 0.36 & 0.26 & 0.09 & 0.063 & 134 & 122 & 56 & 37.1 & 12.5 \\
60 & 4 & 1.8 & 30 & 0.38 & 0.33 & 0.13 & 0.058 & 128 & 135 & 68 & 37.2 & 12.5 \\
70 & 5 & 1.9 & 30 & 0.40 & 0.71 & 0.28 & & 132 & 126 & 60 & 37.2 & 12.5 \\
80 & 6 & 1.7 & 30 & 0.36 & 0.47 & 0.17 & 0.054 & 104 & 133 & 72 & 37.1 & 12.5 \\
88 & 7 & 2.0 & 30 & 0.42 & 0.32 & 0.13 & \multicolumn{1}{c}{102} & 135 & 60 & & \\
90 & Intravenous injection of hexamethonium & $(0.75$ mg. per kg.) \\
100 & 8 & 2.0 & 60 & 0.21 & 0.11 & 0.02 & 0.051 & 74 & 86 & 40 & 37.0 & 12 \\
110 & 9 & 1.7 & 60 & 0.18 & $*$ & & 0.041 & 64 & 80 & 24 & 36.8 & 12 \\
140 & 10 & 1.0 & 90 & 0.07 & 0.08 & 0.006 & 0.039 & 94 & 111 & 7 & 35.6 & 12
\end{tabular}

Exp. 6. Dog $10.1 \mathrm{~kg}$. $\delta$

before

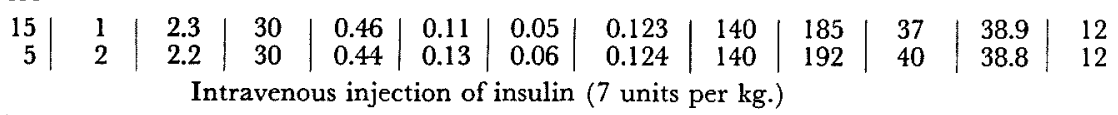

after Intravenous injection of insulin ( 7 units per kg.)

\begin{tabular}{l|r|l|l|l|l|l|l|l|l|l|l|l}
30 & 3 & 2.1 & 30 & 0.42 & 0.54 & 0.23 & 0.060 & 124 & 132 & 59 & 39.0 & 12 \\
60 & 4 & 1.8 & 30 & 0.36 & 0.59 & 0.21 & 0.054 & 132 & 160 & 61 & 38.9 & 12 \\
70 & 5 & 1.8 & 30 & 0.36 & 0.64 & 0.23 & 0.054 & 124 & 175 & 66 & 38.8 & 11.5 \\
80 & 6 & 1.5 & 30 & 0.30 & 0.74 & 0.22 & 0.054 & 118 & 165 & 60 & 38.8 & 11.5 \\
88 & 7 & 1.6 & 30 & 0.32 & 0.79 & 0.25 & 0.053 & 120 & 148 & 42 & & \\
95 & Intravenous injection of hexamethonium & 0.75 mg. per & kg.) \\
105 & 8 & 1.4 & 60 & 0.14 & 0.24 & 0.03 & 0.047 & 88 & 130 & 33 & 38.5 & 12 \\
125 & 9 & 1.8 & 60 & 0.18 & 0.13 & 0.02 & 0.047 & 104 & 136 & 40 & 37.7 & 12 \\
140 & 10 & 1.5 & 60 & 0.15 & 0.16 & 0.02 & 0.048 & 100 & 135 & 26 & 37.4 & 12
\end{tabular}

before

Exp. 7. Dog $14.0 \mathrm{~kg}$.

$$
\begin{array}{r|r|r|l|l|l|l|l|l|l|l|l|l}
15 & 1 & 1.8 & 60 & 0.13 & 0.16 & 0.02 & 0.112 & 146 & 165 & 14 & 38.5 & 14.5 \\
5 & 2 & 1.9 & 60 & 0.14 & 0.15 & 0.02 & 0.114 & 120 & 188 & 16 & 38.2 & 14.5 \\
\text { Intravenous injection of insulin (7 units per kg.) }
\end{array}
$$

\begin{tabular}{|c|c|c|c|c|c|c|c|c|c|c|c|c|}
\hline 30 & 3 & 1.5 & 60 & 0.11 & 0.31 & 0.03 & 0.066 & 112 & 180 & 20 & 38.1 & 14 \\
\hline 60 & 4 & 1.2 & 60 & 0.09 & 0.66 & 0.06 & 0.051 & 94 & 163 & 27 & 38.0 & 14 \\
\hline 70 & 5 & 1.2 & 60 & 0.09 & 0.60 & 0.05 & 0.047 & 94 & 170 & 29 & 37.9 & 14 \\
\hline 80 & 6 & 1.8 & 60 & 0.13 & 0.64 & 0.08 & 0.045 & 98 & 175 & 28 & 37.8 & 14 \\
\hline 88 & 7 & 1.4 & 60 & 0.10 & 0.68 & 0.07 & & 94 & 165 & 30 & & 14 \\
\hline 90 & \multicolumn{12}{|c|}{ Intravenous injection of hexamethonium ( $0.75 \mathrm{mg}$. per kg.) } \\
\hline 100 & 8 & 1.5 & 60 & 0.11 & 0.12 & 0.01 & 0.039 & 70 & 121 & 20 & 37.1 & 14 \\
\hline 110 & 9 & 1.8 & 90 & 0.09 & 0.22 & 0.02 & 0.035 & 72 & 115 & 20 & 36.9 & 14 \\
\hline 120 & 10 & 1.3 & 60 & 0.09 & 0.22 & 0.02 & 0.035 & 74 & 120 & 20 & 36.7 & 14 \\
\hline 150 & 11 & 1.9 & 60 & 0.14 & 0.15 & 0.02 & 0.030 & 82 & 128 & 8 & 36.1 & 14 \\
\hline
\end{tabular}

after 
$0.066 \% 30$ minutes after insulin and it was $0.045 \% 80$ minutes, $0.039 \%$ 100 minutes, $0.035 \% 120$ minutes and $0.030 \% 150$ minutes after insulin.

In this experiment, the initial adrenaline secretion rate was $0.02 \mu \mathrm{g}$. per kg. per minute. On receiving insulin, the rate of secretion was measureed as $0.03 \mu \mathrm{g}$. 30 minutes after insulin, $0.06 \mu \mathrm{g}$. 60 minutes, $0.05 \mu \mathrm{g} .70$ minutes, $0.08 \mu \mathrm{g} .80$ minutes and $0.07 \mu \mathrm{g} .88$ minutes, respectively. Hexamethonium was then injected. The secretion rate of adrenaline was decreased and was measured as $0.01 \mu \mathrm{g}$. per kg. per minute 10 minutes after hexamethonium and $0.02 \mu \mathrm{g} .20,30$ and 60 minutes.

Comparing the results obtained in the hexamethonium-experiments mentioned above with those of the control ones, it is evident that the augmented adrenaline secretion causable by insulin hypoglycemia is suppressed by the application of hexamethonium.

In my previous investigation it was proved that the augmentation of adrenaline secretion causable by acetylcholine was inhibited by hexamethonium. Since acetylcholine acts exclusively peripherally on the adrenal medulla to accelerate the adrenaline secretion, it is sure that in this case the site of action of hexamethonium is the adrenal medulla itself.

Therefore it is reasonable to suppose that in the case of insulin hypoglycemia, which acts through the central mechanism to augment the adrenaline secretion in releasing acetylcholine at the terminals of adrenalinosecretory nerve fibres, hexamethonium suppresses the augmentation of adrenaline secretion also by acting directly on the adrenal medulla.

\section{SUMMARY}

In dogs anesthetized with evipan-sodium, the adrenal venous blood specimens were collected through the lumbar route preparation. The adrenaline content of the specimens was measured colorimetrically by means of the Bloor \& Bullen's arseno-molybdic acid method. The blood sugar content was also determined simultaneously.

Insulin was applied intravenously in a dose of 7 units per $\mathrm{kg}$. of body weight. The adrenaline secretion rate was increased gradually, and from 1 to 2 hours after insulin a definite increase was invariably observed. At the height of augmentation of adrenaline secretion, hexamethonium was injected intravenously in a dose of $0.75 \mathrm{mg}$. per $\mathrm{kg}$. In all cases the secretion rates were cut down to low levels by hexamethonium. It was measured in 3 cases as $0.02-0.04 \mu \mathrm{g}$. per kg. per minute after hexamethonium against $0.13-0.30 \mu \mathrm{g}$. before hexamethonium. In one case, it was $0.01 \mu \mathrm{g}$. against $0.07 \mu \mathrm{g}$.

Thus it is concluded that hexamethonium acts to inhibit the accelerating adrenaline secretion causable by insulin hypoglycemia. 
It is my pleasure to acknowledge the encouragement and advice I have received from Prof. T. Suzuki. I am also grateful to Dr. I. Tanaka for his help given in each of my experiments.

\section{References}

1) Yamashita, K., Jap. J. Physiol., 1956, 6, 279.

2) Schachter, M., J. Physiol., 1951, 115, 206.

3) Satake, Y., Sugawara, T. \& Watanabe, M., Tohoku J. Exp. Med., 1927, 8, 501.

4) Bloor, W. R. \& Bullen, S. S., J. Biol. Chem., 1941, 138, 727. 